\title{
Potencialidad de varias especies para la obtención de biomasa con fines energéticos en el secano mediterráneo de Chile
}

\author{
Potential of some species for biomass energy production in the mediterranean \\ dryland of Chile
}

\author{
Rómulo Santelices-Moya ${ }^{\mathrm{I}}$, Sergio Espinoza-Meza ${ }^{\mathrm{II}}$, \\ Antonio Cabrera-Ariza ${ }^{\mathrm{III}}$, Alejandro Medina-Urbina ${ }^{\mathrm{IV}}$
}

\begin{abstract}
Resumen
En Chile existen aproximadamente 1,8 millones de hectáreas de suelos considerados de secano, con severas restricciones de sitio para el crecimiento de especies agrícolas y forestales. Sin embargo, dichos suelos, pueden ser utilizados y existe un potencial para establecer cultivos energéticos empleando especies forestales especialmente adaptadas a esta condición. El propósito de este trabajo es estimar la superficie potencial para establecer plantaciones energéticas de corta rotación, con especies forestales de bajos requerimientos hídricos, en el secano mediterráneo de la zona central de Chile. Se utilizaron las especies Acacia saligna, Atriplex nummularia, Robinia pseudoacacia, Eucalyptus rudis, Eucalyptus camaldulensis, Eucalyptus occidentalis y Eucalyptus globulus subespecie biscotata, en base a características como rendimiento de biomasa en secano, requerimientos hídricos, tolerancia a heladas y propiedades calorimétricas. El área de estudio corresponde al secano mediterráneo de la Región del Maule en Chile, con una superficie total de 544 mil hectáreas. Los resultados indican que el potencial de cultivo para las siete especies consideradas es de aproximadamente 100.000 ha. Se concluye que el secano mediterráneo de la Región del Maule en Chile presenta condiciones favorables para el establecimiento y desarrollo de plantaciones energéticas con cultivos de corta rotación.
\end{abstract}

Palabras-clave: Bioenergía; Biomasa; Cultivos de corta rotación; Secano

\begin{abstract}
In Chile, there are about 1.8 million hectares of dryland soils with severe site restrictions for agronomic and forest species growth. However, these soils can be used to establish energy crops, using forest species specially adapted to this condition. The purpose of this paper is to estimate the potential land availability in the drylands of central Chile for establishing short rotation forestry crops, with low water consumption species. The species Acacia saligna, Atriplex nummularia, Robinia pseudoacacia, Eucalyptus rudis, Eucalyptus globulus subspecies biscotata, Eucalyptus camaldulensis and Eucalyptus occidentalis, based on characteristics such as dry biomass yield, water requirements, tolerance to frost and calorimetric properties, were evaluated. The study area corresponds to Mediterranean drylands of Maule Region in Chile, with a total area of 544 thousand hectares. The results indicate that the potential for the seven crop species considered is about 100,000 ha. It can be concluded that thousands of land hectares are potentially available for growing short rotation forestry crops in the Mediterranean drylands of central Chile.
\end{abstract}

Keywords: Bioenergy; Biomass; Short rotation crops; Dryland

Ingeniero Forestal, Dr., Centro de Desarrollo para el Secano Interior, Facultad de Ciencias Agrarias y Forestales, Universidad Católica del Maule, Av. San Miguel 3605 Talca-Chile. rsanteli@ucm.cl (ORCID: 0000-0002-5790-8701)

II Ingeniero forestal, Dr., Investigador, Facultad de Ciencias Agrarias y Forestales, Universidad Católica del Maule, Avenida San Miguel 3605 Talca, Chile. espinoza@ucm.cl (ORCID: 0000-0002-6073-2778)

III Ingeniero de Montes, Dr., Vicerrectoría de Investigación y Posgrado, Centro de Desarrollo para el Secano Interior, Facultad de Ciencias Agrarias y Forestales, Universidad Católica del Maule, Av. San Miguel 3605 Talca-Chile. acabrera@ucm.cl (ORCID: 0000-0003-3626-9645)

IV Ingeniero de Ejecución Forestal, MSc., Centro de Desarrollo para el Secano Interior, Universidad Católica del Maule, Av. San Miguel 3605 Talca, Chile. alemedinaur@hotmail.com (ORCID: 0000-0002-8762-6473) 


\section{Introducción}

Chile, al igual que otros países no tiene grandes reservas de petróleo o gas natural, lo que implica que necesita de abastecimiento externo. Este problema nacional justifica el estudio y desarrollo de plantas de energía alternativas, cuya materia prima sea una fuente local. En este último aspecto, la biomasa y la bioenergía tienen un rol fundamental que jugar, especialmente en terrenos que no compitan con la producción agrícola para fines alimentarios, contribuyendo a recuperar suelos degradados junto con servir de sumideros para emisiones de $\mathrm{CO}_{2}$ y que, además, contribuyan con generación de empleos a nivel rural. En este ámbito, el secano interior de la Región del Maule, con una superficie aproximada de 544 mil hectáreas se caracteriza por exhibir condiciones de sitio que si bien resultan marginales para cultivos forestales tradicionales (con rotación mayor a 15 años), posee condiciones adecuadas para el establecimiento de cultivos de corta rotación. Esta área geográfica está asociada a una condición particular que se caracteriza por su pluviometría concentrada en 3 a 4 meses de invierno y un período de sequía de 7 a 9 meses. Cerca del $60 \%$ de las lluvias anuales se concentra durante los 3 meses de invierno, el otoño se presenta con alta probabilidad de lluvia sobre todo en el mes de mayo. En las otras estaciones se reduce la caída pluviométrica anual a un $15 \%$ en primavera y sólo a un $2 \%$ en verano (OSORIO et al., 1995). Este régimen hídrico coincide con los meses en que las temperaturas son poco favorables para el crecimiento vegetal, constituyéndose en una limitante para la producción. Para estas tierras de secano la bioenergía puede ser una alternativa factible a desarrollar. Sin embargo, y a pesar de la creciente demanda energética, no se cuenta con información precisa a nivel comunal o provincial en lo relativo a la obtención sustentable de cantidades suficientes de biomasa con las calidades requeridas para abastecer una planta transformadora, como tampoco en el conocimiento específico de las especies que se podrían explotar en terrenos que no compitan con la producción agrícola para fines alimentarios.

Para que las plantaciones forestales comerciales sean exitosas debe asegurarse que las áreas seleccionadas cumplan con los requerimientos ecológicos propios de las especies que se utilizarán, con ello se logrará que los individuos tengan una adaptación rápida al área donde se establezcan (RUEDA, 1998). Una herramienta que facilita la localización y delimitación de las zonas potenciales para la ubicación de plantaciones forestales comerciales son los Sistemas de Información Geográfica (SIG), (SÁENZ et al., 2000). Un SIG es un sistema de hardware, software y procedimientos diseñados para la captura, administración, manipulación, análisis, modelado y graficado de datos u objetos referenciados espacialmente, que ayudan a resolver problemas complejos de planeación y administración (CHUVIECO, 1996). Con la disponibilidad de los SIG, ha habido varios estudios que involucran el análisis espacial para la determinación de superficie potencial para producción de biomasa (FIORESE; GUARISO, 2010; ABOLINA et al., 2015), resaltando el potencial de esta tecnología en el apoyo a la planificación del abastecimiento de biomasa. Actualmente, en el Noroeste de Europa se utilizan modelos basados en SIG para determinar el potencial de las praderas para abastecer de biomasa en proyectos de generación de energía (HAASE et al., 2016; KETZER et al., 2017).

En Chile se ha generado información acerca del potencial de los desechos del sector forestal para la generación de energía, sabiéndose que en la Región del Maule éstos podrían aportar unos $95 \mathrm{MW}$ de potencia instalada (COMISIÓN NACIONAL DE ENERGÍA, 2008). Para esta región también se conoce la superficie potencial para establecer cultivos energéticos (OFICINA DE ESTUDIOS Y POLÍTICAS AGRARIAS, 2010), sin embargo, no se detalla la superficie por comuna, ni mucho menos se analiza el efecto de la tenencia de la tierra, que incide en la disponibilidad real de tierras. En el caso de las plantaciones con especies del género Eucalyptus se señala que existe un área potencial de 1,8 millones de hectáreas para toda la región. El objetivo del presente trabajo es estimar la superficie potencial del secano interior de la Región del Maule en Chile para establecer cultivos de corta rotación con distintas especies forestales y una especie forrajera. 


\section{Materiales y métodos}

\section{Materiales}

La información empleada para determinar el área de estudio y posteriormente la superficie potencial para cada especie corresponde a una base de datos digital SIG que incorpora información vectorial (curvas de nivel, caminos, cursos de agua, entre otras) generada por el Centro de Desarrollo para el Secano Interior de la Universidad Católica del Maule en el año 2005. Se consideró, además, una cobertura SIG correspondiente a la ocupación del suelo según Catastro de Bosque Nativo, elaborada por CONAF-CONAMA a escala 1:20.000 el año 1999.

\section{Área de estudio}

El estudio se realizó en la zona del secano mediterráneo de la Región de Maule, Chile, comúnmente conocido como "secano interior". El área de estudio se encuentra en la zona límite de las comunas de Rauco y Curicó por el norte, y Cauquenes por el sur en la Región del Maule y está ubicada entre los paralelos $71^{\circ}$ y $73^{\circ}$ de latitud sur y $35^{\circ}$ y $36^{\circ}$ de longitud oeste abarcando una superficie de 544.000 hectáreas (Figura 1). La zona posee un clima mediterráneo cálido con precipitaciones promedio de $800 \mathrm{~mm}$, además tiene un período seco de cinco meses entre noviembre y marzo, ciclo que presenta las máximas temperaturas medias de $19^{\circ} \mathrm{C}$ y extremas de $30^{\circ} \mathrm{C}$ en los valles y cuencas interiores, mientras que en invierno las temperaturas mínimas alcanzan un promedio de 7C (SANTIBÁÑEZ; URIBE, 1993). Esta condición se da producto del efecto "sombra de lluvias" que se produce en la vertiente oriental de la Cordillera de la Costa, es decir, una disminución de la precipitación en la pendiente de barlovento. La vegetación dominante corresponde al Matorral Espinoso, comunidad caracterizada por la presencia de arbustos de la estepa de Acacia caven Mol., denominado comúnmente espino, asociado a un abundante matorral esclerófilo en los sectores menos secos entre los que se destacan Maytenus boaria Mol., Quillaja saponaria Mol., Lithrea caustica (Mol.) Hook. et Arn., Peumus boldus Mol., Baccharis linearis (Ruiz \&Pav.) Pers. y Crinodendron patagua Mol., entre otros.

Figura 1 - Área de estudio. Secano interior de la Región del Maule en Chile.

Figure 1 - Study area. Mediterranean interior drylands of Maule Region in Chile.

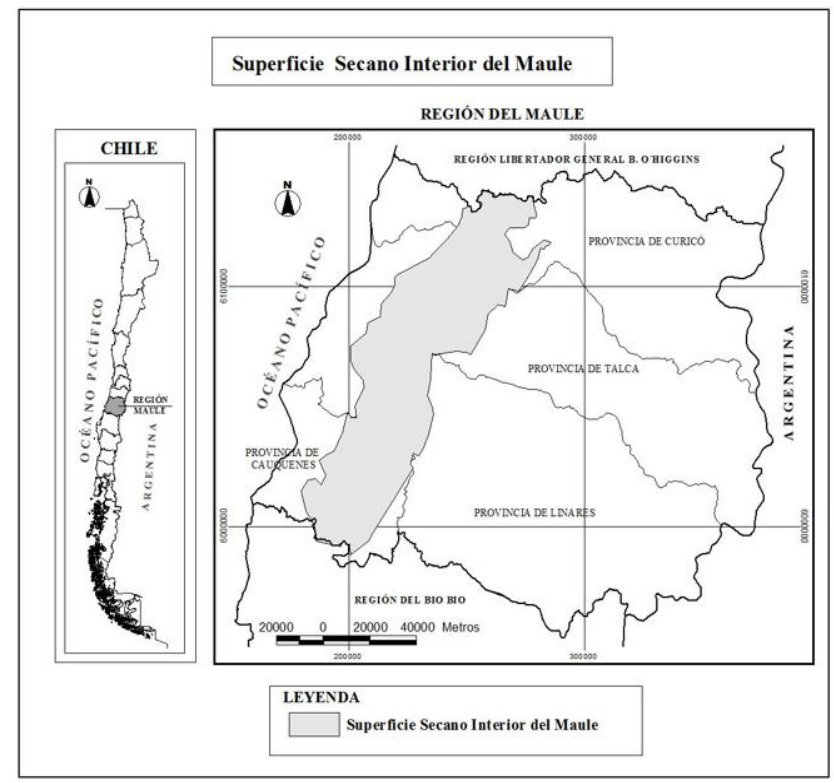

Fuente: Autores (2014) 


\section{Determinación de las variables físicas y ambientales del terreno para la} delimitación de las áreas potenciales

Una vez definido el área de estudio, se procedió a elaborar monografías de los requerimientos ecofisiológicos (suelo y clima) para las especies Acacia saligna (Labill.) H.L. Wendl., Atriplex nummularia Lindl., Robinia pseudoacacia L., Eucalyptus rudis Endl., Eucalyptus occidentalis Endl., Eucalyptus camaldulensis Dehnh. y Eucalyptus globulus Labill. subespecie biscotata. Con la información obtenida se confeccionó una tabla con los requerimientos ecológicos para cada una de las especies del estudio (Tabla 1).

\section{Estimación de áreas potenciales}

La estimación de las áreas potenciales para cada una de las especies en estudio se realizó a partir de los requerimientos ambientales mencionados en la Tabla 1 y considerando solamente suelos sin uso o con matorral esclerófilo abierto o muy abierto (i.e. con cobertura menor a $10 \%$ según lo establece la legislación chilena) y sin restricciones para su corta. Para esto se utilizaron las capas temáticas de clima y suelo, las que se sobrepusieron en el SIG ArcGis versión 9.3 mediante el comando OVERLAY y seleccionando la operación multiplicación, para obtener las áreas potenciales por especie en formato raster. Luego, a través del comando AREA se calculó la superficie para cada especie y posteriormente se elaboró la cartografía para estimar la superficie potencial para establecer cultivos energéticos en el secano interior de la Región del Maule.

Tabla 1 - Requerimientos climáticos y edáficos de las siete especies.

Table 1 - Site requirements for the potential seven species.

\begin{tabular}{lccccc}
\hline \multicolumn{1}{c}{ Especie } & $\begin{array}{c}\mathbf{P P} \\
(\mathbf{m m})\end{array}$ & $\begin{array}{c}\mathbf{T}^{\mathbf{0}} \mathbf{M a x} \\
\left({ }^{\circ} \mathbf{C}\right)\end{array}$ & $\begin{array}{c}\mathbf{T}^{\circ} \mathbf{M i n} \\
\left({ }^{\circ} \mathbf{C}\right)\end{array}$ & $\begin{array}{c}\mathbf{T}^{\mathbf{0}} \text { media } \\
\left({ }^{\circ} \mathbf{C}\right)\end{array}$ & $\begin{array}{c}\text { Textura } \\
\text { Suelo }\end{array}$ \\
\hline $\begin{array}{l}\text { Eucalyptus globulus spp. } \\
\text { biscotata }\end{array}$ & $600-1.600$ & $20-28$ & -1 a -6 & $5-17$ & Muy fina \\
$\begin{array}{l}\text { Eucalyptus rudis } \\
\text { Eucalyptus occidentalis }\end{array}$ & $450-1.010$ & $26-30$ & -1 a 3 & $13-17$ & Muy fina \\
$\begin{array}{l}\text { Eucalyptus camaldulensis } \\
\text { Acacia saligna }\end{array}$ & $300-910$ & $23-34$ & $2-9$ & $12-18$ & Media \\
Acacia nummularia & $300-6000$ & $22-41$ & $0-14$ & $18-19$ & Moderadamente fina \\
Robinia pseudoacacia & $230-650$ & $32-37$ & -8 & $15-24$ & Moderadamente fina \\
\hline
\end{tabular}

Fonte: (NEWMANN et al., 2006; BENELL et al., 2009; CLARKE; MCLEOD; VERCOE, 2009; HOBBS; BENELL, 2009; HOBBS et al., 2009a; 2009b)

$\mathrm{PP}=$ precipitación media anual; $\mathrm{T}^{\mathrm{o}} \mathrm{Max}=$ Temperatura máxima del mes más cálido; $\mathrm{T}^{\mathrm{o}}$ Min= Temperatura mínima del mes más frío; $\mathrm{T}^{\mathrm{o}}$ Media= Temperatura media anual

\section{Resultados y discusión}

Se generaron mapas que muestran las áreas potenciales para la ubicación de plantaciones con fines energéticos en el secano mediterráneo de la Región del Maule en la zona central de Chile. Los resultados indican que el potencial de cultivo para las especies consideradas en el presente estudio bordea las 100.000 ha. En el caso de las especies Eucalyptus rudis, Eucalyptus globulus subespecie biscotata y Acacia nummularia la superficie potencial bordea las 17 mil hectáreas. Para Acacia saligna la superficie 
es cercana a las 100.000 ha y para Eucalyptus camaldulensis, la superficie también es cercana a las 100.000 ha (Figura 2), en tanto que para Robinia pseudoacacia ésta sólo alcanza las 1.125 hectáreas. Finalmente, para Eucalyptus occidentalis, la superficie es también de 1.125 hectáreas, debido principalmente a las restricciones en cuanto a la precipitación (deben ser mayores de $600 \mathrm{~mm}$ ) y textura del suelo (Figura 3).

\section{Figura 2 - Superficie potencial de Eucalyptus camaldulensis en el secano interior de la Región del Maule en Chile.}

Figure 2 - Potential area for Eucalyptus camaldulensis in the Mediterranean interior drylands of Maule Region in Chile.

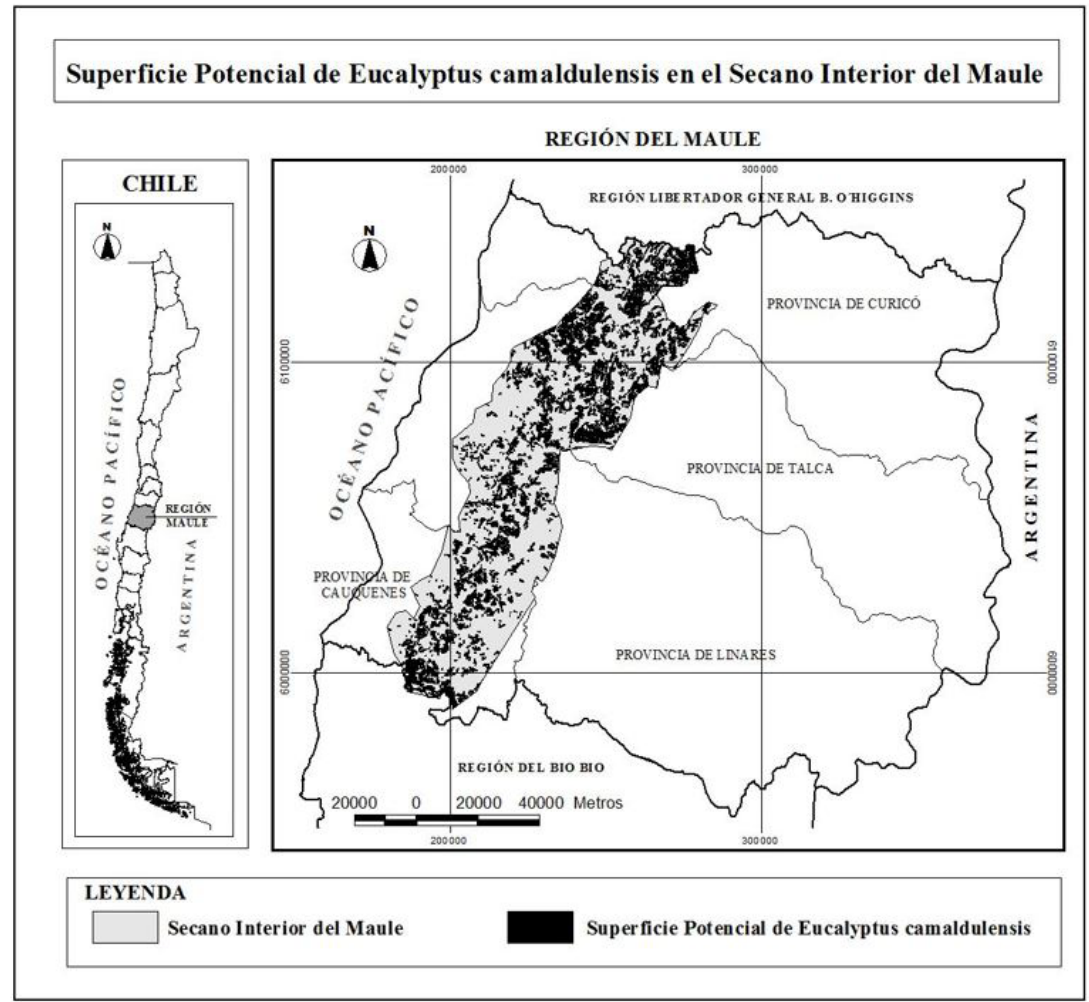

Fuente: Autores (2014)

Se puede observar que dos de las especies con el mayor potencial de cultivo en el secano interior de la Región del Maule en Chile son Eucalyptus camaldulensis (Figuras 2) y Acacia saligna. En el caso de Eucalyptus camaldulensis, esta corresponde a una especie tolerante a la sequía, a las altas temperaturas, a las inundaciones periódicas y a una moderada salinidad. En Australia se ha usado ampliamente para recuperar suelos degradados (escombreras de minas y terrenos inundables) y es la especie más plantada en zonas mediterráneas. Se presenta en suelos arcillosos y también arenosos (CLARKE; MCLEOD; VERCOE, 2009; HOBBS; BENNELL, 2009). La especie se ha cultivado en distintas zonas de secano del mundo con resultados promisorios. En el secano de Nicaragua, Van den Broek y Van Wijk (1998) y Van den Broek et al. (2001) han reportado productividades de 13 ton $\mathrm{MS} \mathrm{ha}^{-1} \mathrm{año}^{-1}$. En el caso de Acacia saligna, especie conocida por su amplia aptitud para ser establecida en ambientes áridos (HNATIUK; MASLIN, 1988; MCDONALD; MASLIN, 2000), ha recibido especial atención en zonas de secano de Australia por su potencial para la obtención de forraje para animales y biomasa para generación de energía, teniendo un alto potencial de crecimiento en zonas con precipitaciones en el rango de $300-600 \mathrm{~mm}$ año-1 $(\mathrm{HOBBS}$ et al., 2009a). En el mediterráneo del sur de Túnez la especie se puede encontrar creciendo en sitios con precipitaciones de $196 \mathrm{~mm}$ año ${ }^{-1}$ (JEDDI; CORTINA; CHAIEB, 2009). La especie también ha sido cultivada en zonas extremadamente áridas como el Desierto de Negev en Israel, con precipitaciones de $96 \mathrm{~mm}_{\text {año-1 }}$ (ZEGADA-LIZARAZU et al., 2007), en la India, donde las condiciones de sitio son extremas 
con temperaturas superiores a los $46^{\circ} \mathrm{C}$ en el día y $0^{\circ} \mathrm{C}$ por la noche durante el verano, precipitaciones que no sobrepasan los $300 \mathrm{~mm}$ año-1 y suelos arenosos con una baja capacidad de retención de agua (TEWARI et al., 2002) y en Pakistán, donde el monto de precipitaciones alcanza los $350 \mathrm{~mm}$ año-1 la temperatura máxima llega hasta los $48^{\circ} \mathrm{C}$ y la evapotranspiración bordea los $1.553 \mathrm{~m}$ año-1 (MAHMOOD et al., 2003). Esto confirma el potencial de estas dos especies para ser establecidas en el secano mediterráneo de Chile, que posee un monto de precipitaciones entre los $600-700 \mathrm{~mm}^{-}$año- $^{-}$ 1. Sin embargo, un aspecto que es de vital importancia en terrenos de secano es el manejo aplicado a las plantaciones. Es necesario definir una silvicultura específica para cultivos de corta rotación, especialmente en terrenos ambientalmente frágiles de secano, con el propósito de generar y aumentar el conocimiento específico sobre este tipo de cultivos, en aspectos tales como silvicultura preventiva de incendios forestales, especies y procedencias adecuadas, densidades y marcos de plantación óptimas, reciclaje de nutrientes, propiedades físicas y químicas de la biomasa, sistemas de cosecha y logística, además de aspectos como consumo de agua, susceptibilidad a plagas y enfermedades, entre otros, a modo de producir biomasa de manera económicamente eficiente y ambientalmente sustentable. En este sentido, se ha probado que especies del género Eucalyptus, manejadas con silvicultura intensiva pueden crecer en áreas con menos de $600 \mathrm{~mm}^{2} \mathrm{no}^{-1}$ de precipitación y suelos delgados con baja fertilidad (BOARDMAN; SIMPSON, 1981; FREDERICK et al., 1985; HILLIS, 1990; DALIANIS et al., 1996; GUO et al., 2002; 2006; FAÚNDEZ, 2003; PÉREZ et al., 2006). Esto ha llevado a señalar que las plantaciones de Eucalyptus son una alternativa viable para el abastecimiento de biomasa en proyectos energéticos tanto en Estados Unidos (GONZÁLEZ et al., 2011), como en el Reino Unido (LESLIE et al., 2012).

Otras especies con un potencial promisorio en el secano mediterráneo de Chile son Eucalyptus rudis y Acacia nummularia. En el caso de Acacia nummularia, especie endémica de la zona árida del este y centro de Australia, se desarrolla en sitios donde el rango de precipitación está en los 150-600 mm año-1 (HOBBS et al., 2009b). También se ha establecido en zonas áridas de otras partes del mundo como Botswana (AGANGA; MTHETHO; TSHWENYANE, 2003), Israel (ARIELI et al., 1989), Siria (JONES; AROUS, 2000), Egipto (ABOU El NASR et al., 1997), y también en la zona norte de Chile, donde el monto de precipitaciones no supera los $100 \mathrm{~mm}$ año-1 (ALONSO, 1990). Otro aspecto destacable de la especie es su alta tolerancia a las heladas, llegando a tolerar hasta $-10^{\circ} \mathrm{C}$ (MAIDEN, 1894), lo que resalta su potencial de uso en el secano mediterráneo de Chile. En el caso de Eucalyptus rudis, si bien ha sido utilizado principalmente para combatir el problema de la salinidad del suelo en el oeste de Australia, también hay experiencias exitosas en zonas de secano donde los montos de precipitación son bajos y los suelos son muy degradados (HOBBS et al., 2009b).

Se debe señalar que el área potencial obtenida para las distintas especies no compite con terrenos destinados a la agricultura, sino que se estaría sobreponiendo en un $100 \%$ con zonas desprovistas de vegetación o con coberturas arbóreas muy bajas (e.g. matorral abierto, matorral muy abierto). A pesar de que en la superficie potencial de cultivo no existen zonas destinadas a la conservación de recursos naturales, las actividades planificadas con el objetivo de establecer proyectos de plantaciones con fines energéticos con las especies propuestas, deben ser cuidadosamente proyectadas. En el área propuesta los cuerpos de agua existentes son formaciones de menor tamaño, y considerando su condición de tierras de secano con la consiguiente restricción hídrica, deben ser aislados y mantenidos sin la presencia de este tipo de cultivos, dando así cumplimiento, además, a la normativa legal vigente en el país. Especialmente hay que considerar que en los primeros años de crecimien to las plantaciones del género Eucalyptus consumen una importante cantidad de agua. Sin embargo, esta información es todavía muy general en términos del potencial real debido a que en dicho espacio se configuran una serie de factores socioeconómicos y de competitividad que reducen la posibilidad de aprovechamiento de dichas áreas. Por ejemplo, se desconoce el modelo de tenencia de la tierra y su impacto en la disponibilidad real de tierras para implementar proyectos de generación de energía a base de biomasa. Esto genera el desafío futuro de analizar el modelo de tenencia de la tierra en este sector, junto con la determinación de la real disponibilidad de sus propietarios para participar en este tipo de proyectos. Es necesario, introducir al modelo inicial de potencialidad, coberturas adicionales sobre uso actual del suelo (áreas de uso agrícola, ganadero, forestal, etc.) y ocupación 
del territorio (concesiones diversas o derechos adquiridos sobre la tierra) para así poder estimar el potencial real en superficie con el que cuenta esta región, a modo de generar información para que los actores involucrados en el negocio puedan tomar sus decisiones de inversión. De acuerdo a la realidad de los pequeños productores en esta área, que en su mayoría (más del 90\%) manejan una agricultura de subsistencia, se considera necesario pensar en un sistema asociado como modelo de negocios para la introducción del cultivo de Eucalyptus y las otras especies consideradas en esta investigación, dando opción al agricultor de aprovechar cosechas trienales.

\section{Figura 3 - Superficie potencial de Eucalyptus occidentalis en el secano interior de la Región del Maule en Chile.}

Figure 3 - Potential area for Eucalyptus occidentalis in the Mediterranean interior drylands of Maule Region in Chile.

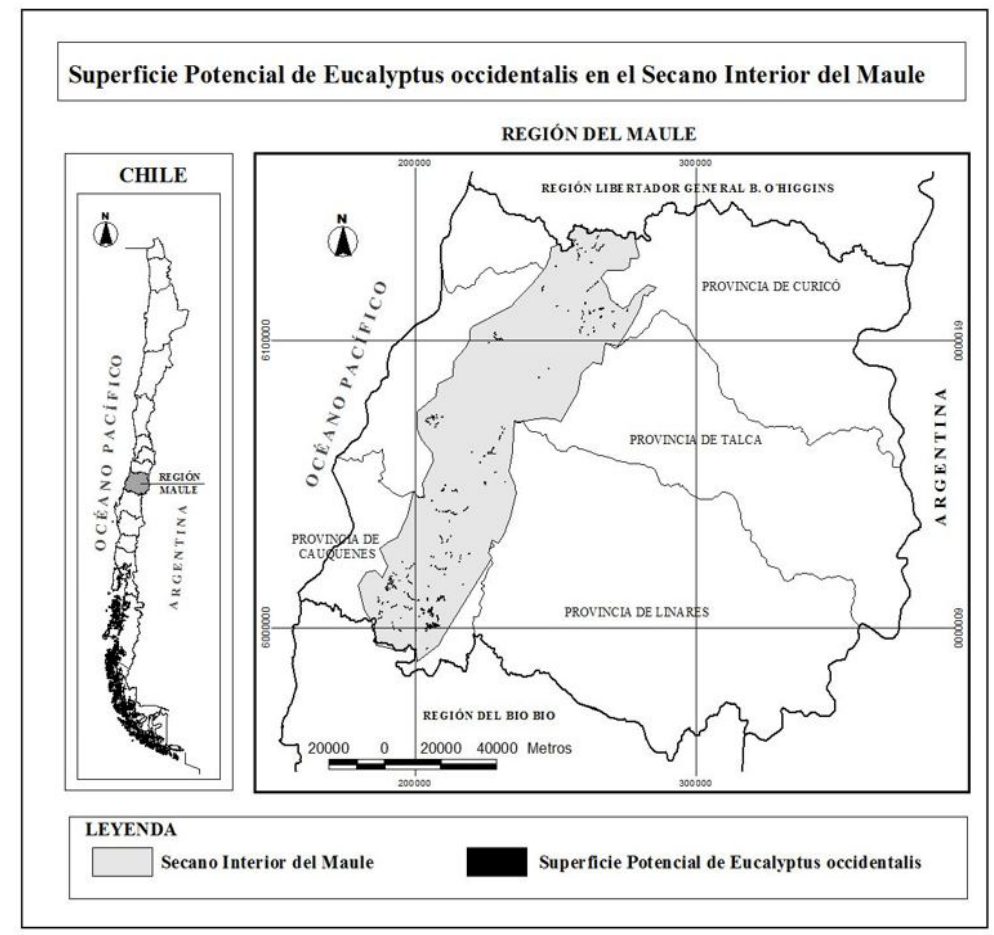

Fuente: Autores (2014)

\section{Conclusiones}

El secano interior de la Región del Maule presenta una superficie aproximada de 100.000 ha con condiciones biofísicas aceptables para el establecimiento de plantaciones con fines energéticos y generar biomasa para abastecer plantas generadoras de energía eléctrica y/o térmica. La mayor parte de esta superficie está desprovista de vegetación, erosionada o cubierta con matorral esclerófilo (principalmente estepa de Acacia caven, donde predominan formaciones arbustivas con una cobertura de copa inferior al $10 \%$, ubicados en terrenos con pendientes entre $0 \%$ a $30 \%$, que no se considerarían como bosque. Sin embargo, hay aspectos en los que se debe profundizar el estudio a futuro. Estos son 1) la necesidad de instalar una red de ensayos de campo que permita evaluar la supervivencia y crecimiento de las especies propuestas, 2) incorporar el modelo de tenencia de la tierra, para determinar la real disponibilidad de terrenos, y 3) incorporar el uso actual del suelo, para determinar el cambio de uso al que ha estado sujeto el territorio. 


\section{Referencias}

ABOLINA, E. et al. GIS based agricultural land availability assessment for the establishment of short rotation woody crops in Latvia. Biomass and Bioenergy, Oxford, v. 72, p. 263-272, 2015.

ABOU El NASR, H. M. et al. Value of processed saltbush and acacia shrubs as sheep fodders under the arid conditions of Egypt. Small Ruminant Research, Amsterdam, v. 24, n. 1, p. 15-20, 1997.

AGANGA, A. A.; MTHETHO, J. K.; TSHWENYANE, S. Atriplex nummularia (Old Man Saltbush): a potential forage crop for arid regions of Botswana. Pakistan Journal of Nutrition, Faisalabad, v. 2, n. 2, p. 72-75, 2003.

ALONSO, J. L. Reforestation of arid and semi-arid zones in Chile. Agriculture, Ecosystems and Environment, Amsterdam, v. 33, n. 2, p. 111-127, 1990.

ARIELI, A. et al. The effect of feeding saltbush and sodium chloride on energy metabolism in sheep. Animal Production, Cambridge, v. 49, n. 3, p. 451-457, 1989.

BENNELL, M. et al. Evaluating agroforestry species and industries for lower rainfall regions of southeastern Australia. Flora Search 1a. Canberra: RIRDC, 2009. 232 p.

BOARDMAN, R.; SIMPSON, J. Fertilization to optimize productivity. In: TURVEY, N. D. (Ed.). Productivity in perpetuity. Melbourne: Australian Forestry Council, 1981. p. 303-317.

CHUVIECO, E. Fundamentos de teledetección espacial. 3. ed. Madrid: RIALP, 1996. 568 p.

ClARKe, B.; MCLEOD, I.; VERCOE, T. Trees for Farm Forestry: 22 Promising Species. Canberra: RIRDC, 2009. 239 p.

COMISIÓN NACIONAL DE ENERGÍA (Chile). Potencial de generación eléctrica por residuos del manejo forestal en Chile. Santiago: CNE; GTZ, 2008. 54 p.

DALIANIS, C. et al. Very short rotation and dense eucalypt plantations for energy. In: PROCEEDINGS OF THE NINTH EUROPEAN BIOENERGY CONFERENCE ON BIOMASS FOR ENERGY AND THE ENVIRONMENT, 6., 2010, Copenhagen. Proceedings... New York: Pergamon, 1996. p. 7.

FAÚNDEZ, P. Potential costs of four short-rotation silvicultural regimes used for the production of energy. Biomass and Bioenergy, Oxford, v. 24, p. 373-380, 2003.

FIORESE, G., GUARISO, G. A GIS-based approach to evaluate biomass potential from energy crops at regional scale. Environmental Modelling \& Software, Oxford, v. 25, n. 6, p. 702-711, 2010.

FREDERICK, D. et al. Dry matter content and nutrient distribution in an age series of Eucalyptus regnans plantations in New Zealand. New Zealand Journal of Forestry Science, New York, v. 15, n. 2, p. 158-179, 1985.

GONZÁLEZ, R. etal. Exploring the potential of Eucalyptus for energy production in the Southern United States: financial analysis of delivered biomass. Part I. Biomass and Bioenergy, Oxford, v. 35, n. 2, p. 755-766, 2011.

GUO, L. et al. Biomass production and nutrient cycling in Eucalyptus short rotation energy forests in New Zealand. I: biomass and nutrient accumulation. Bioresource Technology, Oxford, v. 85, p. 273-283, 2002.

GUO, L. et al. Biomass production and nutrient cycling in Eucalyptus short rotation energy forests in New Zealand. II. Litter fall and nutrient return. Biomass and Bioenergy, Oxford, v. 30, p. 393-404, 2006.

HAASE, M. et al. GIS-based assessment of sustainable crop residue potentials in European 
regions. Biomass and Bioenergy, Oxford, v. 86, p. 156-171, 2016.

HILLIS, W. Fast growing eucalyptus and some of their characteristics. In: WERNER, D.; MULLER, P. (Ed.). Fast growing trees and nitrogen fixing trees. Stuttgart; New York: Gustav Fischer Verlag, 1990. p. 184-193.

HNATIUK, R. J.; MASLIN, B. R. Phytogeography of acacia in Australia in relation to climate and species-richness. Australian Journal of Botany, Melbourne, v. 36, n. 4, p. 361-383, 1988.

HOBBS, T. J.; BENNELL, M. Agroforestry species profiles for lower rainfall regions of southeastern Australia. Canberra: RIRDC, 2009. 242 p. (Flora Search, 1).

HOBBS T. J. et al. Developing species for woody biomass crops in lower rainfall southern Australia. Canberra: RIRDC, 2009a. 242 p. (Flora Search, 3).

HOBBS T. J. et al. Potential agroforestry species and regional industries for lower rainfall southern Australia. Canberra: RIRDC, 2009b. 138 p. (Flora Search, 2).

JEDDI, K.; CORTINA, J.; CHAIEB, M. Acacia salicina, Pinus halepensis and Eucalyptus occidentalis improve soil surface conditions in arid southern Tunisia. Journal of Arid Environments, London, v. 73, n. 11, p. 1005-1013, 2009.

JONES, M. J.; AROUS, Z. Barley-Salt-Bush intercropping for sustainable feed production in a dry mediterranean steppe environment. Journal of Agronomy and Crop Science, Berlin, v. 184, p. $253-260,2000$.

KETZER, D. et al. Assessment of sustainable Grassland biomass potentials for energy supply in Northwest Europe. Biomass and Bioenergy, Oxford, v. 100, p. 39-51, 2017.

LESLIE, A. D. et al. The potential for Eucalyptus as a wood fuel in the UK. Applied Energy, Oxford, v. 89, n. 1, p. 176-182, 2012.

MAHMOOD, K. et al. Genetic variation in Eucalyptus camaldulensis Dehnh. for growth and stem straightness in a provenance-family trial on saltland in Pakistan. Forest Ecology and Management, Amsterdam, v. 176, n. 1/3, p. 405-416, 2003.

MAIDEN, J. H. Useful Australian Plants - No. 9 An Old Man Salt-Bush (Atriplex nummularia, Lindl.). Agricultural Gazette of New South Wales, Sydney, v. 4, p. 209-213, 1984.

MCDONALD, M. W.; MASLIN, B. R. Taxonomic revision of the Salwoods: Acacia aulacocarpa Cunn. ex Benth. and its allies (Leguminosae: Mimosoideae: section Juliflorae). Australian Systematic Botany, Melbourne, v. 13, n. 1, p. 21-78, 2000.

NEUMANN, C. et al. Flora Search - Developing broad scale commercial revegetation industries in low rainfall regions of southern Australia. In: VEG FUTURE 06: THE CONFERENCE IN THE FIELD, 3., 2006, Albury. Actas... Victoria: Greening Australia, 2006. 11 p.

OFICINA DE ESTUDIOS Y POLÍTICAS AGRARIAS (Chile). Mapas de zonificación de aptitud productiva del territorio nacional de especies vegetales con potencial de producción de biocombustibles. Santiago: ODEPA, 2010. 235 p.

OSORIO, J. et al. Caracterización agroecológica de la VII Región del Maule. Talca: Escuela de Agronomía, Facultad de Recursos Naturales, Universidad de Talca, 1995. 50 p.

PÉREZ, S. et al. Energy potential of waste from 10 forest species in the North of Spain (Cantabria). Bioresource Technology, Oxford, v. 99, n. 14, p. 6339-6345, 2006.

RUEDA, S. A. Áreas potenciales para plantaciones forestales de 11 especies de pino en Jalisco. 1998. 109 h. Disertación (Tesis de Maestría) - Universidad de Guadalajara, Guadalajara, 1998.

SÁENZ, R. et al. Regionalización de áreas potenciales para plantaciones forestales en el oriente de Michoacán. In: PRIMER CONGRESO NACIONAL DE REFORESTACIÓN, 11., 2000, 
Montecillo. Actas... México: SEMARNAP; PRONARE, 2000. 6 p.

SANTIBAÑEZ, F.; URIBE, J. Atlas agroclimático de Chile. VI, VII, VIII y IX Regiones. Santiago: Laboratorio de Agroclimatología, Facultad de Ciencias Agrarias y Forestales, Universidad de Chile, 1993. 73 p.

TEWARI, V. et al. Growth and yield functions for irrigated plantations of Eucalyptus camaldulensis in the hot desert of India. Bioresource Technology, Oxford, v. 85, n. 2, p. 137-146. 2002.

VAN DEN BROEK, R. et al. The energy crop growth model SILVA: description and application to eucalyptus plantations in Nicaragua. Biomass and Bioenergy, Oxford, v. 21, n. 5, p. 335-349, 2001.

VAN DEN BROEK, R.; VAN WIJK, A. Electricidad a partir de eucalipto y bagazo en ingenios azucareros en Nicaragua. Costos, aspectos macroeconómicos y medioambientales. Rome: FAO, 1998.

ZEGADA-LIZARAZU, W. et al. Above and below ground development of Acacia saligna shrubs grown under different irrigation frequencies in an arid environment. Plant Soil, Dordrecht, v. 297, p. 157-169, 2007. 